\title{
Strengthening and stretching for rheumatoid arthritis of the hand (SARAH): design of a randomised controlled trial of a hand and upper limb exercise intervention - ISRCTN89936343
}

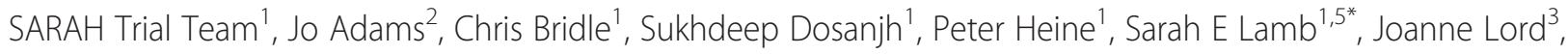
Christopher McConkey ${ }^{1}$, Vivien Nichols ${ }^{1}$, Francine Toye ${ }^{4}$, Martin R Underwood ${ }^{1}$, Mark A Williams ${ }^{1}$ and Esther M Williamson ${ }^{1}$

\begin{abstract}
Background: Rheumatoid Arthritis (RA) commonly affects the hands and wrists with inflammation, deformity, pain, weakness and restricted mobility leading to reduced function. The effectiveness of exercise for RA hands is uncertain, although evidence from small scale studies is promising. The Strengthening And Stretching for Rheumatoid Arthritis of the Hand (SARAH) trial is a pragmatic, multi-centre randomised controlled trial evaluating the clinical and cost effectiveness of adding an optimised exercise programme for hands and upper limbs to best practice usual care for patients with RA.

Methods/design: 480 participants with problematic RA hands will be recruited through 17 NHS trusts. Treatments will be provided by physiotherapists and occupational therapists. Participants will be individually randomised to receive either best practice usual care (joint protection advice, general exercise advice, functional splinting and assistive devices) or best practice usual care supplemented with an individualised exercise programme of strengthening and stretching exercises. The study assessors will be blinded to treatment allocation and will follow participants up at four and 12 months. The primary outcome measure is the Hand function subscale of the Michigan Hand Outcome Questionnaire, and secondary outcomes include hand and wrist impairment measures, quality of life, and resource use. Economic and qualitative studies will also be carried out in parallel.

Discussion: This paper describes the design and development of a trial protocol of a complex intervention study based in therapy out-patient departments. The findings will provide evidence to support or refute the use of an optimised exercise programme for RA of the hand in addition to best practice usual care.
\end{abstract}

Trial registration: Current Controlled Trials ISRCTN89936343

Keywords: Randomised controlled trial, Rheumatoid arthritis, Exercise, Hand, Rehabilitation

\footnotetext{
* Correspondence: s.lamb@warwick.ac.uk

${ }^{1}$ Warwick Clinical Trials Unit, Warwick Medical School, University of Warwick, Coventry CV4 7AL, UK

${ }^{5}$ Kadoorie Critical Care Research Centre, John Radcliffe Hospital, Oxford OX3 9DU, UK

Full list of author information is available at the end of the article
} 


\section{Background}

Rheumatoid arthritis (RA) is the most common inflammatory polyarthritis [1]. It is a chronic unpredictable disorder that can cause persistent joint pain, joint damage and long-term disability (especially in the hands and feet). The prevalence of RA is $1.16 \%$ in women and $0.44 \%$ in men, increasing with age to $5 \%$ in those aged over 55 [1]. Five years after diagnosis, $40 \%$ of people with RA have relatively normal function (13\% in remission), $44 \%$ have mild to moderate disability, and $16 \%$ have marked functional disability [2]. Particular problems for the hands and wrists are inflammation, deformity, pain, weakness and restricted mobility resulting in loss of function [3].

Although there is no cure for the disease, there are increasingly effective drug treatments that can reduce the impact of the disease, notably disease-modifying anti-rheumatic drugs (DMARDs). The overall goals of management are to prevent or control joint damage, maximise function and decrease pain [4]. All current UK clinical guidelines for the management of RA recommend the use of physiotherapy (PT) and occupational therapy (OT) as an adjunct to drug treatment [5,6]. The three most common components of PT/OT for RA hands are exercise therapy, joint protection advice and provision of functional splinting and assistive devices [7].

A systematic review [8] of six randomized controlled trials (RCTs) of the effectiveness of exercise programmes in RA for the whole body concluded that dynamic exercise (aerobic capacity and/or muscle strength training) was effective in improving muscular endurance and strength, without detrimental effects on disease activity or pain. The number of RCTs that have specifically investigated the effects of exercise on RA hands is limited to five small studies $(\mathrm{n}=55,100,67,52$ and 57 patients respectively) with mostly short term follow up of a few months [9-13]. Each of these studies demonstrated small improvements in hand strength or function using exercise, with no increase in joint swelling, pain or disease activity. The long term effectiveness of exercise for RA hands has not been established.

The economic cost of RA is thought to be substantial for both the individual patient and society as a whole $[4,14]$. The highest costs are associated with those patients that have poor and declining function early on in their disease [15].

\section{Aims}

This paper describes the trial protocol of a large pragmatic randomised controlled trial to evaluate the clinical and cost effectiveness of adding an optimised exercise programme for hands and upper limbs to best practice usual care for patients with RA. An additional aim is to describe, qualitatively, the experience of participants with a particular emphasis on patient expectation, exercise behaviours, and reasons for adherence/ non-adherence.

\section{Methods}

Trial design

The SARAH trial is a pragmatic, multi-centre randomised controlled trial (Figure 1).

\section{Participants}

480 participants will be recruited from direct referrals from Rheumatology clinics and from those referred to PT/OT clinics. 17 NHS trusts in England will recruit participants.

In addition to the recruitment of newly referred patients, a number of the Rheumatology and some of the PT/OT participating departments have a review register of 'chronic' patients, periodically called in for review appointments. Provided that these patients meet the selection criteria described below, they will be approached to participate in the trial.

\section{Selection criteria}

All patients with Rheumatoid Arthritis meeting the American College of Rheumatology clinical and immunological criteria [4], with pain and dysfunction of the hands and/or wrist joints who are either not on a disease modifying medication (DMARD), or who have been on a stable DMARD regimen for three months or more, will be included in the trial. Those fulfilling any of the following criteria will be excluded:

i. Aged less than 18 years.

ii. Patients who have experienced upper limb joint surgery, or fracture, in the previous six months.

iii. Patients on a waiting list for upper limb orthopaedic surgery.

iv. Patients who are pregnant.

Patients will be asked to give written informed consent according to principles of Good Clinical Practice and the Declaration of Helsinki [16]. At the time of consent, outcome assessors will collect baseline measures.

\section{Randomisation}

Randomisation to the exercise programme or usual care will be via a central telephone randomisation service at Warwick Clinical Trials Unit, University of Warwick. The randomisation schedule will be prepared by the trial statistician $(\mathrm{CM})$. Randomisation will be stratified by centre using a variable block size. 


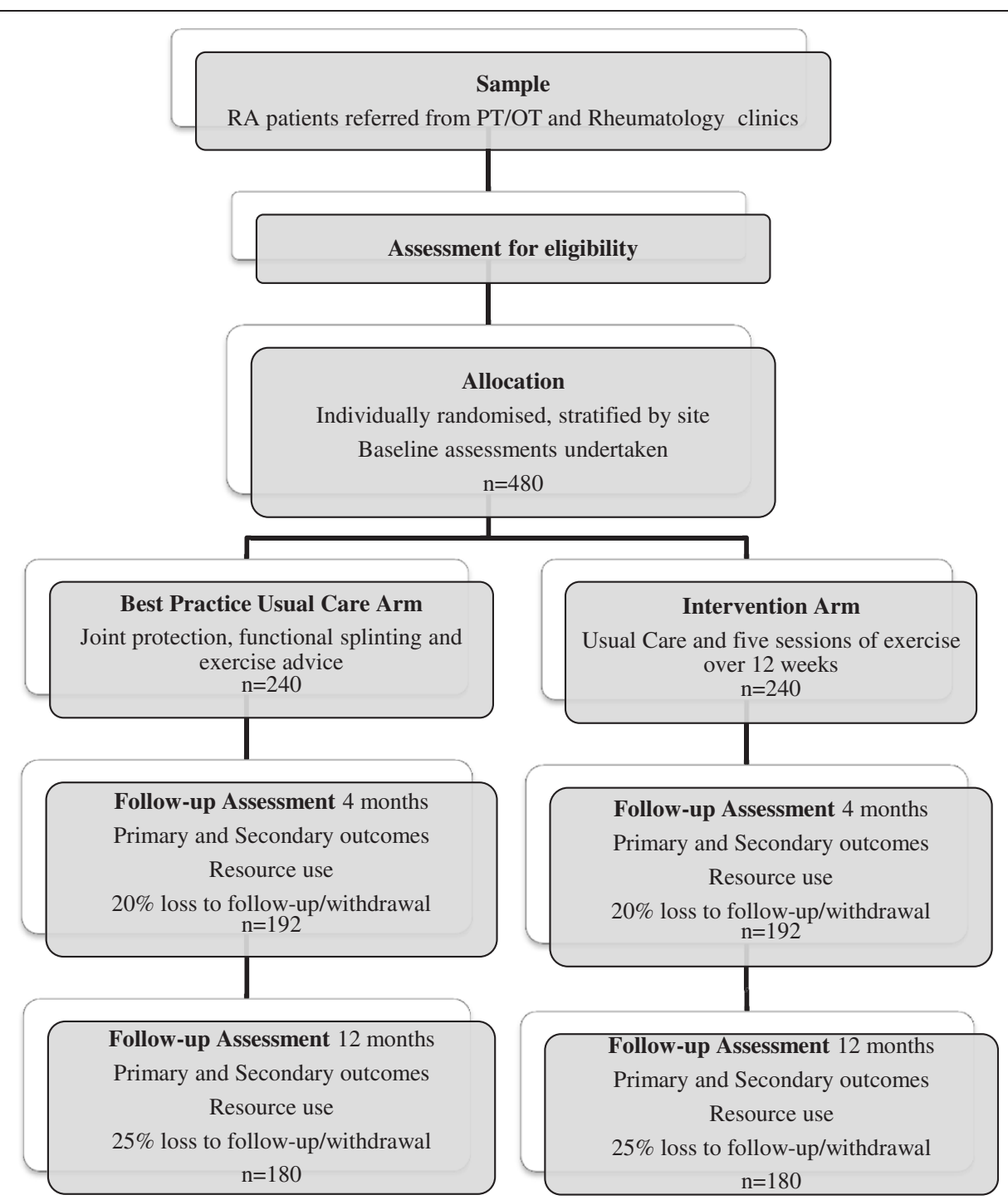

Figure 1 Flowchart of study design.

\section{Allocation concealment}

Eligibility checks will be performed, and consent for randomisation taken. The research clinician will then telephone the randomisation service, and only once the patient is registered in the trial, will the random allocation be generated. Hence allocation will be concealed.

\section{Blinding}

The outcome assessor will be blind to the group allocation of the participant and will be independent of intervention delivery. Participants will be requested not to disclose group allocation to the outcome assessor. If an outcome assessor is unblinded, this will be recorded. All assessors will be asked to guess which allocation they think the participant has been given at both follow-up time points. The patients and therapists providing the treatment cannot be blinded to the group allocation.

\section{Baseline assessment}

After participants have been assessed for eligibility and consent has been gained, baseline assessment will be carried out. Questionnaires will be completed whilst the outcome assessor conducts randomisation followed by a physical assessment (both participant and assessor will be unaware of allocation at this appointment). The baseline measures are summarised in Table 1. A Research Clinic Questionnaire will record demographic details (age, sex, date of RA diagnosis, ethnicity, marital status, hand dominance) and the Michigan Hand outcomes Questionnaire (MHQ) [17], The MHQ has shown to be a reliable, valid and responsive measure for an RA population [18-20] and contains six domains (1) overall hand function, (2) Activities of Daily Living (ADL), (3) pain, (4) work performance, (5) aesthetics, and (6) patient satisfaction. Scores range from 0 to 100 , with higher scores 
Table 1 Summary of measures to be collected

\begin{tabular}{|c|c|c|c|}
\hline Domain & Data source & $\begin{array}{l}\text { Measures - Instrument (Scale - high value is better score unless } \\
\text { specified) }\end{array}$ & $\begin{array}{l}\text { Time } \\
\text { points }\end{array}$ \\
\hline \multirow[t]{2}{*}{ Function } & \multirow[t]{2}{*}{$\begin{array}{l}\text { Research Clinic Questionnaire } \\
\text { (participant reported) }\end{array}$} & $\begin{array}{l}\text { Michigan Hand Outcomes Questionnaire (MHQ) - overall Hand function score } \\
(0-100)\end{array}$ & \multirow[t]{2}{*}{$0,4,12$} \\
\hline & & Michigan Hand Outcomes Questionnaire (MHQ)- overall score (0-100) & \\
\hline \multirow[t]{2}{*}{ Pain } & \multirow[t]{2}{*}{ Research Clinic Questionnaire } & Pain sub-scale of MHQ (0-100; high score is worse) & \multirow[t]{2}{*}{$0,4,12$} \\
\hline & & 'Troublesomeness' rating (0-20; high score is worse) & \\
\hline \multirow[t]{6}{*}{ Impairment } & \multirow{6}{*}{$\begin{array}{l}\text { Research Clinic Examination } \\
\text { (performed by outcome assessor) }\end{array}$} & Joint deformity (MCPJ only) - goniometer (Degrees; high score is worse) & \multirow[t]{6}{*}{$0,4,12$} \\
\hline & & Wrist range of motion (flexion/extension) - goniometer (Degrees) & \\
\hline & & $\begin{array}{l}\text { Finger range of motion (combined flexion and combined extension) - Ruler } \\
\text { (mm; high score is worse for combined flexion) }\end{array}$ & \\
\hline & & Thumb opposition range of motion - observation (0-10) & \\
\hline & & Dexterity - timed 9 hole peg test (Seconds; high score is worse) & \\
\hline & & Grip and Pinch Strength - dynamometer (Newtons) & \\
\hline \multirow[t]{2}{*}{ Disease Activity } & \multirow[t]{2}{*}{$\begin{array}{l}\text { Medical Records Research Clinic } \\
\text { Examination }\end{array}$} & $\begin{array}{l}\text { Erythrocyte sedimentation rate (ESR - mm/h) and/or C-Reactive protein (CRP - } \\
\mathrm{mg} / \mathrm{l}) \text { blood test }\end{array}$ & \multirow{2}{*}{$0,4,12$} \\
\hline & & $\begin{array}{l}\text { Hand and wrist joint tenderness and swelling count - examination (0-22; high } \\
\text { score is worse) }\end{array}$ & \\
\hline \multirow{2}{*}{$\begin{array}{l}\text { Health-related } \\
\text { Quality of Life }\end{array}$} & \multirow[t]{2}{*}{ Research Clinic Questionnaire } & SF-12(0-100) & \multirow[t]{2}{*}{$0,4,12$} \\
\hline & & EuroQol EQ-5D (health utility)(0-1) & \\
\hline Self-efficacy & Research Clinic Questionnaire & 7 item questionnaire & $0,4,12$ \\
\hline \multirow[t]{2}{*}{ Satisfaction } & \multirow[t]{2}{*}{ Research Clinic Questionnaire } & Treatment satisfaction item & \multirow[t]{2}{*}{4,12} \\
\hline & & Satisfaction sub-scale of MHQ (0-100) & \\
\hline Global Change & Research Clinic Questionnaire & Participant-rated global change question(7 point Likert scale) & 4,12 \\
\hline Adherence & Research Clinic Questionnaire & 5 item questionnaire & $0,4,12$ \\
\hline Economics & Research Clinic Questionnaire & Resource use questionnaire & 4,12 \\
\hline
\end{tabular}

indicating better performance, except for the pain scale. For the pain scale, a higher score indicates more pain.

The questionnaire will also contain measures of pain troublesomeness [21], self-efficacy [22], the EuroQol EQ5D [23], the 12 item short form health survey (SF-12) [24], health economics-related questions (employment status, sick days in past month due to RA in wrists/ hands, benefits, highest educational qualification, household income) and treatment preference. Blood test results (CRP, ESR, serum rheumatoid factor) and current medication (prescribed and as required in last 7 days) will be taken from hospital and prescription records. The outcome assessor will be present to answer any questions regarding the measures but will be trained not to influence the participant's responses.

Following completion of the case report form, a physical assessment will be performed in a standardised order and standardised positions. This will include the measurement of joint deformity (MCP ulnar/radial deviation in maximum pronation, where ulnar deviation is recorded as a positive value) and active range of motion (wrist flexion and extension from the neutral position with a goniometer [25], combined finger flexion according to Ellis and Bruton [26], combined finger extension and thumb opposition according to Kapandji [27]). A modified swollen and tender joint count (22 joints of hand and wrist [28]) will be taken, along with a test of upper limb dexterity (Nine-hole peg test according to Mathiowetz et al. [29]). Finally, two forms of grip strength (full-hand and tripod pinch will be measured using the MIE Digital Grip Analyser [30]. The standard test position recommended by the American Society of Hand Therapists will be used [31]. Patients will be sat in a straight-backed chair without arm rests, feet flat on floor, the shoulder of the assessed limb will be relaxed by the side, the elbow flexed to 90 degrees, the wrist will be extended and in ulnar deviation between 0 and 15 degrees and the forearm rotated to neutral pronation/ supination. The mean of three maximal three-second grips will be calculated for each hand, with 60 second rests between repetitions.

\section{Interventions}

The rationale and protocol for the interventions are described in a separate paper [32]. All interventions are delivered by PTs or OTs experienced in the treatment of hand and rheumatology conditions. The therapists will be independent of the recruitment and randomisation procedures and will attend a training session by the trial team and receive a training and reference manual as well 
as on-going support and guidance regarding the intervention to ensure quality standards are met. Therapists will be permitted to deliver both the experimental and control interventions, and each treatment session will be recorded in a treatment log. Contamination will be minimized through monitoring of treatment logs completed at each session, quality assurance visits to each therapist at the beginning of their time delivering interventions on the trial and also limitation of additional therapy materials (Patient exercise booklets, therapeutic putty, Thera-band ${ }^{\circledR}$ resistive band and hand exercisers) sufficient to only cover participants randomised to the experimental arm of the study. The aim is for all treatments to be completed within four months of the baseline assessment. Figure 2 provides an overview of the interventions.

a. Control intervention - Best Practice Usual Care only

Participants randomised to the control intervention will have between one and three sessions of outpatient therapy with a maximum total contact time of one and a half hours. Treatment will include the provision of joint protection information, splinting, assistive devices and

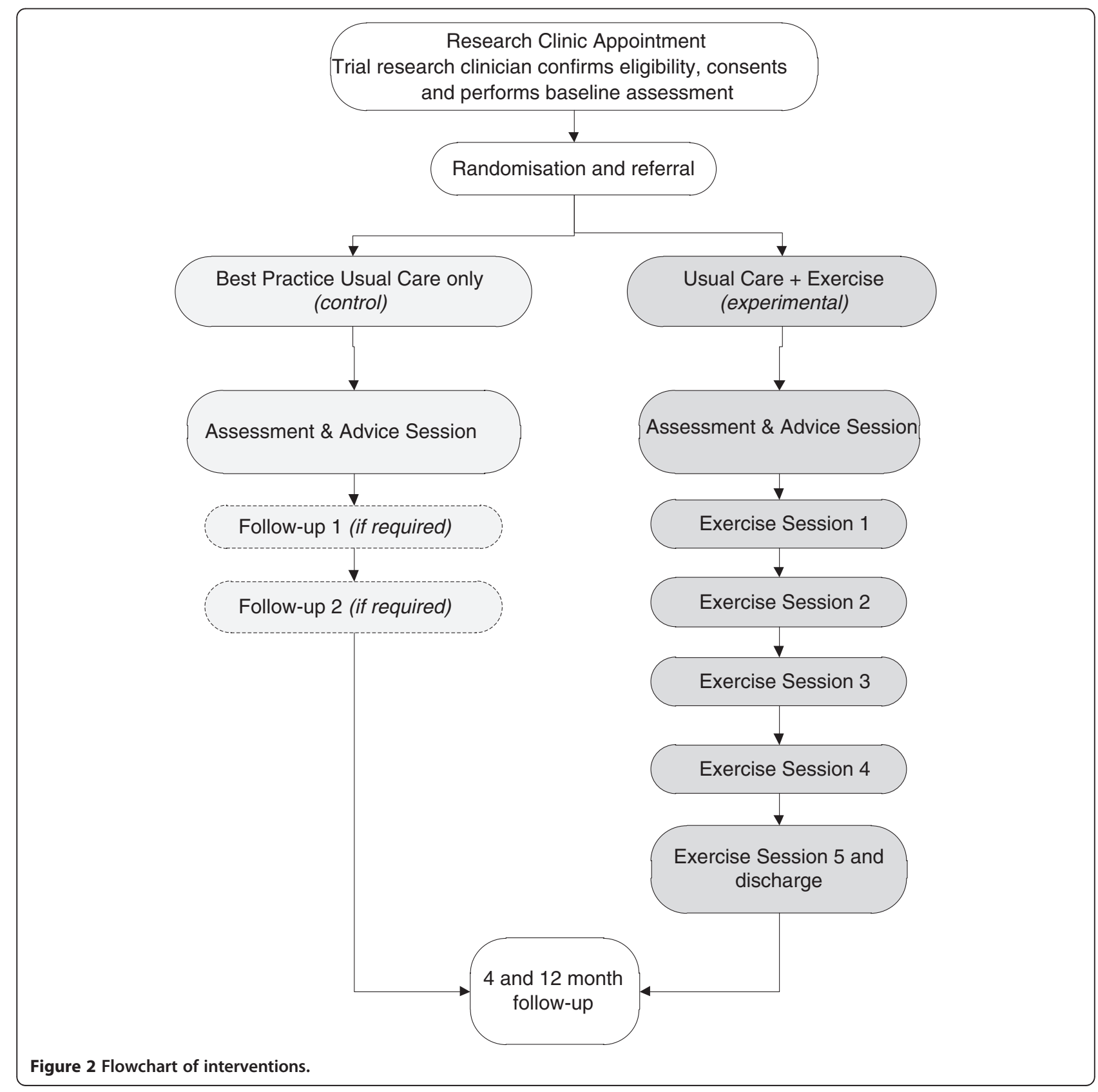


other general advice as required. The number of sessions will depend on quantity of advice and education required and/or for review of splinting/assistive devices. This will be determined by the stage and severity of the disease. The choice of treatments was made using two principles; first, there is evidence that the treatments are effective for hand dysfunction in RA [33], and second, the treatments are consistent with current clinical guidelines $[5,6]$. Both control and experimental interventions were developed using focus group meetings with stake holder clinicians from across the UK.

b. Experimental intervention - Best Practice Usual Care supplemented with an optimised exercise programme

Participants randomised to the exercise programme will receive the usual care package plus an additional five sessions with a therapist over a 12 week period. The aim will be to increase hand function using exercises to stretch and strengthen the muscles and tendons, as well as mobilise the joints of the hand and wrist and improve dexterity. This will be supported by a Home Exercise Programme (reinforced by a behavioural activation approach and exercise diary) to be performed daily. This number of contacts, spread over this period, allows maximum progression of the intensity of exercise and sufficient time for a physiological response in the neuromuscular system to significantly improve function [34]. The intervention will use a standardised protocol of progression and, if necessary, regression of exercise intensity. A modified Borg scale of perceived exertion [35] will be used to regulate the intensity of resistance exercise. The programme is based on the existing evidence base, a professional consensus of UK PT/OTs and a programme that has some evidence of shortterm effectiveness [36]. Adherence with the exercise programme is vital to ensure the dosage required to strengthen muscle and improve mobility is achieved. Evidence based strategies to maximise adherence will be incorporated into each treatment session, including effective goal setting and action planning $[37,38]$.

Treatment fidelity will be evaluated by recording content of sessions on treatment logs and the number of sessions attended by participants in both arms of the trial. Quality assurance checks will be made by the clinical research fellow who will observe treatment sessions for all therapists. We will also evaluate whether participants have progressed exercises within the exercise arm using information from the treatment logs and exercise diaries.

\section{Other treatments}

Participants may seek other forms of treatment during the follow-up period. Additional treatments, including contacts with their GP or other health professional, changes in types of medication, use of physical treatments or alternative therapies, will be recorded as a treatment outcome.

\section{Follow-up data collection}

Follow-up data collection will be by face-to-face clinical assessment at four and 12 months. Where face-to-face clinic assessment is not possible, postal and telephone data collection methods will be used to obtain core data. The outcome measures have been described in the baseline assessment section and are detailed in Table 1 . The primary outcome measure will be the 12 month Michigan Hand Outcome Questionnaire (MHQ) overall hand function subscale score.

\section{Sample size}

A standardised mean effect size of 0.3 is reported to represent a clinically important difference in hand function in this group [39]. A previous small study using a similar intervention found a mean benefit of 0.7 in the AIMS2 with a standard deviation of 1.81 and a standardised effect size of 0.39 [36]. This suggests that in this larger, more pragmatic multi-centre trial, a standardised effect size of 0.3 in a similar function score of the MHQ is realistic and meaningful. To show this difference with $80 \%$ power at the $5 \%$ significance level, we require data on at least 352 participants (using SAS procedure GLMPOWER). Assuming a worst case scenario of $25 \%$ loss to follow-up, this would require 470 participants to be recruited initially.

Over 15 months we expect 1,200 people with hand RA to be referred to our participating centres. If half of these are assessed for study entry and $80 \%$ of these join the study, we will have 480 participants $(1,200 * 0.5 * 0.8)$. This is our target sample size (Figure 1). The assumptions underlying the sample size calculation will be monitored by the Data Monitoring Committee.

\section{Statistical analysis}

Patients will be analysed in the treatment group to which they were randomised, regardless of the treatment that they actually received [40]. The trial will be reported to CONSORT guidance and standards [41], and all estimates will be reported $95 \%$ confidence intervals. Demographic, clinical characteristics and baseline measurements will be presented to compare the trial groups and generalisability to clinical settings.

\section{Primary outcome}

The difference between the intervention and best practice usual care groups in mean MHQ overall hand function subscale score at 12 months will be analysed by a linear model, adjusted for the baseline score. Adjustment 
will be made for centre, sex and age. Further adjustment by disease activity, medication and other patient characteristics will be considered if there are imbalances between the intervention groups.

\section{Secondary outcomes}

Differences between groups for MHQ overall hand function score at 4 months, overall MHQ score at 4 and 12 months, measures of pain (sub-scale of MHQ and 'troublesomeness'), impairment measures, self-efficacy, joint counts, and quality of life (SF-12 and EQ-5D) scores will be analysed in a similar manner to the primary outcome measure.

Analyses will also take account of baseline drug regimens (no DMARD, single DMARD, combination DMARD or biologic DMARD) and disease duration ( $<$ or $>5$ years since diagnosis). The impact of therapist effects will be explored by including a random therapist effect nested within centre in the various models. The effect of missing data will be investigated using multiple imputation analysis.

Complier-average causal effect (CACE) will be estimated using an instrumental variable method [42]. Statistical analyses will be performed using SAS V9.2 software.

\section{Economic evaluation}

The economic analysis will include a 'within-trial' costutility analysis, and modelling to estimate longer-term costs and health effects if justified by the clinical results.

For the within-trial analysis, costs incurred and Quality-Adjusted Life Years (QALYs) attained over the twelve month study period will be estimated at the individual patient level. The EuroQol (EQ-5D) will be used to generate individuals' utility scores at 0,4 and 12 months (using the UK Social Tariff [43]), and QALYs will then be calculated over this period using an areaunder-the-curve approach. Similarly, self-reported resource use data collected at 4 and 12 months will be used to estimate individuals' hand RA related health and social care costs over the 12 month study period. The cost of the exercise intervention will be estimated from PT and OT records of time spent with patients, and of any consumables used. Costs will be estimated from an NHS and Personal Social Service perspective (as recommended by NICE [44]), as well as from a broader societal perspective (including patient expenditure). Unit costs for publicly funded services will be taken from standard national sources [45], costs of private services will be obtained directly from patients. As for clinical outcomes, missing resource use and QALY data will be estimated by multiple imputation.

A regression approach will then be used to estimate between-group differences in mean costs and QALYs, adjusting for centre, sex, age and other baseline differences between the study groups. An Incremental Cost-Effectiveness Ratio (ICER), the additional cost per additional QALY gained with the exercise programme compared with best usual practice alone, will be calculated if appropriate. Interaction terms will also be used to investigate possible treatment moderators and hence to identify patient subgroups for whom treatment costeffectiveness is predictably different: age, sex, disease activity, medication group, duration of disease, or other relevant patient characteristics.

The twelve-month time horizon of the within-trial analysis is quite limited. It is possible that extrapolation of costs and QALYs beyond this time might lead to different conclusions about cost-effectiveness. For example, if participants randomised to the exercise programme had greater quality of life at twelve months than those randomised to usual care alone, but that this health gain came at a relatively high cost, then it is possible that further accumulation of quality of life gains and/or cost savings after the first year could bring the ICER below the $£ 20,000$ to $£ 30,000$ per QALY gained threshold that is usually considered to be cost-effective in the UK [46]. If so, we will use decision-analytic modelling with probabilistic sensitivity analysis to extrapolate expected costs and health effects.

\section{Qualitative study}

The qualitative study will help to provide a picture of issues facing patients with hand problems as a result of RA who participate in the experimental intervention arm of the study. Semi-structured interviews will be conducted by a researcher experienced in the design, collection and analysis of qualitative data. Specific topics covered will be living with RA, exercising with RA (including adherence issues), and participant experience of the SARAH trial.

We will interview enough participants to ensure we are confident that theoretical sufficiency will be achieved [47]. Therefore, we are aiming to interview up to 20 participants randomised to receive the exercise intervention. Sampling will be purposive to enable recruitment of patients who report benefitting and not benefitting from the exercise programme.

Interviews will be conducted following four and 12 month follow-up appointments either in hospitals or patient homes. The development of the interview schedule will be iterative and the questions asked may develop and change as the interviews are conducted and analysed [48].

All interviews will be recorded and then transcribed for analysis. Interpretative Phenomenological Analysis (IPA) will be used [49]. A feature of IPA is that the first steps of analysis begin early in the research process with 
initial data coding and is simultaneous to data collection. Initial analysis of each interview will be carried out as soon after its completion as possible following the guidelines set out by Smith et al. [50].

One in five of the interviews will be coded independently by a $2^{\text {nd }}$ member of the research team with experience of qualitative research (FT) to provide additional knowledge by giving a different perspective on the coding [51]. The research team will discuss the development of themes as the research progresses, once again, with the aim of providing a different perspective and enhancing the development of themes. Participants will be given a pseudonym to be used in any reports related to the study, with the option to choose their own pseudonym.

\section{Ethics committee approval}

The SARAH trial was approved by the Oxford C Multicentre Research Ethics (MREC 08/H0606/47) Committee and by the Research and Development department of each participating centre.

\section{Discussion}

Impairment and dysfunction of hands and upper limbs are key concerns for patients with RA and optimal conservative management has yet to be defined and documented. This pragmatic randomised controlled trial investigates whether there is additional benefit of an optimised exercise programme for hands and upper limbs when added to best practice usual care for this group of patients.

A strength of this study is that the exercise programme will be individually tailored to each patient, reflecting clinical practice, and is designed to ensure a sufficient dose of exercise is delivered. Strategies to maximise adherence to the exercise programme are an addition to an already promising intervention. The intervention is designed to fit with the usual constraints of NHS provision.

There is also methodological rigour in the design, range of validated outcome measures and mixed methods utilised that will help to understand underlying mechanisms and key patient perspectives. Long term follow-up and the large sample size will allow for a comprehensive clinical effectiveness analysis. The parallel health economics analysis will enable evaluation of costeffectiveness of treatments which are particularly pertinent in the current economic climate.

\section{Competing interests}

The authors declare that they have no competing interests.

\section{Authors' contributions}

All authors critically revised the manuscript for important intellectual content and approved the final manuscript. JA participated in the conception, design and conduct of the study, obtaining funding and will participate in interpretation of data. CB developed the behavioural activation sections of the intervention and participated in obtaining funding. SD co-ordinated the administration of the study and acquisition of data, administrative and material support. PH participated in design and conduct of the study, intervention development and will participate in interpretation of data. SEL is chief investigator, participated in conception and design of the study, initial drafting of manuscript, obtaining funding, and will participate in analysis and interpretation of data. $J$ participated in the design of the study, drafting of the health economics section of the manuscript and will oversee the health economics analysis. CM participated in the design of the study, obtaining funding, drafting of the statistical analysis section of the manuscript and will conduct the statistical analyses. VN participated in the design and data collection for the qualitative study, drafting of the qualitative section of the manuscript and will conduct the qualitative study analysis. FT participated in the design and analysis of the qualitative study. MRU participated in the conception, design and conduct of the study, obtaining funding, supervision and will participate in interpretation of data. MAW participated in the design and conduct of the study, lead drafting of the manuscript, and will participate in analysis and interpretation of data. EMW led the design and data collection for the qualitative study and will conduct the qualitative study analysis.

\section{Data Monitoring Committee}

Mr Ed Juszczak, Prof Paul Dieppe, Dr Helen Frost.

\section{Trial Steering Committee}

Prof Alison Hammond (Chair), Dr Chris Deighton, Dr Chris McCarthy, Prof Sallie Lamb, Dr Mark Williams, Mr John Wright (User representative).

\section{Acknowledgements}

SARAH Trial team

Chief Investigator: Prof SE Lamb

Co-investigators: Dr J Adams, Prof MR Underwood, Dr C Bridle, C McConkey, Dr J Lord.

Trial lead: Dr MA Williams

Trial co-ordination/Administration: Dr S Dosanjh, S Lowe, A Campbell, L Rattigan

Research Fellows/Associates: P Heine, Dr EM Williamson, V Nichols

Trial statistician: C McConkey

Health economists: Dr J Lord, C Crossan, Dr M Dritsaki, Dr M Glover

Local Principal Investigators: Lynda Myshrall (UHCW NHS Trust), Joanne

Newbold (George Eliot Hospital NHS Trust), Dr Chris Marguerie (South

Warwickshire Hospital NHS Trust), Prof Ashok Rai (Worcester Acute Hospitals NHS Trust), Ann Birch (Wrightington, Wigan \& Leigh NHS Trust), Sarah Bradley (Poole Hospital NHS Trust), Peter Webster (Royal Bournemouth NHS Trust), Sandi Derham (Bath Royal National Hospital for Rheumatic Diseases), Dr Richard Hull (Portsmouth Hospitals NHS Trust), Kevin Spear (Basingstoke \& North Hampshire Hospital NHS Trust), Sarah Wastell (Dorset NHS Trust), Christina McLeod (Winchester \& Eastleigh Healthcare NHS Trust), Dr Karen Barker (Nuffield Orthopaedic Centre NHS Trust), Lorraine Kendall (Bognor Regis War Memorial Hospital), Nicola Bassett-Burr (Western Sussex Hospitals NHS Trust) Dr Arvind Sinha (Solihull Hospital), Nicola Clague-Baker (Leicester Royal Infirmary), Sue Kennedy (Royal Derby Hospital).

Research Clinicians (recruitment and data collection): Olivia Neely, Catherine Gibson, Karen Hotchkiss, Frances Chilton, Jessica Thrush, Catherine MinnsLowe, Ann Birch, Linda Webber, Nicola Clague-Baker, Andrew Robinson, James Portelli, Sue Kennedy, Kevin Spear, Sandi Derham, Dr Jenny Lewis, Sarah Bradley, Julie Cottrell, Paula White, Carole Frosdick, Jennifer Wilson, Nicola Bassett-Burr, Maggie Walsh.

Intervention therapists (delivery of treatments): Lynda Myshrall, Jane Tooby, Cherry Steinberg, Mary Grant, Roslyn Handley, Fiona Jones, Clare Pheasant, Kate Hynes, Sue Kelly (UHCW NHS Trust); Joanne Newbold, Sally Thurgarland (George Eliot Hospital NHS Trust); Jane Dickenson, Lucy Mann (South Warwickshire Hospital NHS Trust); Alison Hinton, Rachel Chapman, Sunita Farmah, Collette James, Janice Wiltshire, Jane Simons (Worcester Acute Hospitals NHS Trust); Jane Martindale, Susan Hesketh, Alison Gerrard (Wrightington, Wigan \& Leigh NHS Trust); Kirsty Bancroft, Corinna Cheng (Poole Hospital NHS Trust); Caroline Wood (Royal Bournemouth NHS Trust); Lisa Small, Karen Coales, Helen Ibbunson, Anne Bonsall (Bath Royal National Hospital for Rheumatic Diseases); Caroline Mountain, Jonathan Gibbons, Esther Mavurah, Hannah Susans (Portsmouth Hospitals NHS Trust); Nicola Spear, Becky Shaylor, Leon Ghulam (Basingstoke \& North Hampshire Hospital 
NHS Trust); Christina McLeod, Sapphire Patterson, Jane Vadher (Winchester \& Eastleigh Healthcare NHS Trust); Sue Gosling, Lizelle Sander-Danby, Jon Room, Aimee Fenn, Anne Richards, Pam Clarke, Gill Rowbotham, Nicky Nolan (Nuffield Orthopaedic Centre NHS Trust); Lorraine Kendall (Bognor Regis War Memorial Hospital); Claire Charnley (Solihull Hospital); Laura Richardson, Kate Wakefield (Leicester Royal Infirmary); Victoria Jansen, Liz Radbourne, Julie Tougher (Royal Derby Hospital).

\section{Funding}

The SARAH trial is funded by the National Institute of Health Research Health Technology Assessment Programme (NIHR HTA), project number 07/32/05. This project benefited from facilities funded through Birmingham Science City Translational Medicine Clinical Research and Infrastructure Trials Platform, with support from Advantage West Midlands (AWM).

\section{Author details}

${ }^{1}$ Warwick Clinical Trials Unit, Warwick Medical School, University of Warwick, Coventry CV4 7AL, UK. 'Faculty of Health Sciences, University of Southampton, Highfield, Southampton, Hampshire SO17 1BJ, UK. ${ }^{3}$ Health Economics Research Group, Brunel University, Uxbridge, Middlesex UB8 3PH, UK. ${ }^{4}$ Nuffield Orthopaedic Centre, Windmill Rd, Headington, Oxford, Oxfordshire OX3 7HE, UK. Kadoorie Critical Care Research Centre, John Radcliffe Hospital, Oxford OX3 9DU, UK

Received: 9 October 2012 Accepted: 2 November 2012

Published: 24 November 2012

\section{References}

1. Symmons D, Turner G, Webb R, Asten P, Barrett E, Lunt M, Scott D, Silman $A$ : The prevalence of rheumatoid arthritis in the United Kingdom: new estimates for a new century. Rheumatology (Oxford) 2002, 41(7):793-800.

2. Young A, Dixey J, Cox N, Davies P, Devlin J, Emery P, Gallivan S, Gough A, James $D$, Prouse $P$, et al: How does functional disability in early rheumatoid arthritis (RA) affect patients and their lives? Results of 5 years of follow-up in 732 patients from the Early RA Study (ERAS). Rheumatology (Oxford) 2000, 39(6):603-611.

3. Adams J, Burridge J, Mullee M, Hammond A, Cooper C: Correlation between upper limb functional ability and structural hand impairment in an early rheumatoid population. Clin Rehabil 2004, 18(4):405-413.

4. American College Of Rheumatology: Guidelines for the management of rheumatoid arthritis: 2002 Update. Arthritis Rheum 2002, 46(2):328-346.

5. Deighton C, O'Mahony R, Tosh J, Turner C, Rudolf M, Group GD: Management of rheumatoid arthritis: summary of NICE guidance. BMJ 2009, 338:b702.

6. Luqmani R, Hennell S, Estrach C, Basher D, Birrell F, Bosworth A, Burke F, Callaghan C, Candal-Couto J, Fokke C, et al: British society for rheumatology and British health professionals in rheumatology guideline for the management of rheumatoid arthritis (after the first 2 years). Rheumatology (Oxford) 2009, 48(4):436-439.

7. Hammond A: Rehabilitation in rheumatoid arthritis: a critical review. Musculoskeletal Care 2004, 2(3):135-151.

8. Van den Ende C, Vliet Vlieland T, Munneke M, Hazes J: Dynamic exercise therapy in rheumatoid arthritis: a systematic review. Br J Rheumatol 1998, 37(6):677-687.

9. Brighton S, Lubbe J, van der Merwe $C$ : The effect of a long-term exercise programme on the rheumatoid hand. Br J Rheumatol 1993, 32(5):392-395.

10. Buljina A, Taljanovic M, Avdic D, Hunter T: Physical and exercise therapy for treatment of the rheumatoid hand. Arthritis Rheum 2001 45(4):392-397.

11. Dellhag B, Wollersjo I, Bjelle A: Effect of active hand exercise and wax bath treatment in rheumatoid arthritis patients. Arthritis Care Res 1992, 5(2):87-92.

12. Hoenig H, Groff G, Pratt K, Goldberg E, Franck W: A randomized controlled trial of home exercise on the rheumatoid hand. J Rheumatol 1993 20(5):785-789.

13. O'Brien A, Jones P, Mullis R, Mulherin D, Dziedzic K: Conservative hand therapy treatments in rheumatoid arthritis-a randomized controlled trial. Rheumatology (Oxford) 2006, 45(5):577-583.

14. Cooper NJ: Economic burden of rheumatoid arthritis: a systematic review. Rheumatology (Oxford) 2000, 39(1):28-33.
15. Yelin $E$, Wanke LA: An assessment of the annual and long-term direct costs of rheumatoid arthritis: the impact of poor function and functional decline. Arthritis Rheum 1999, 42(6):1209-1218

16. Association WM: World Medical Association Declaration of Helsinki. Ethical principles for medical research involving human subjects. Nurs Ethics 2002, 9(1):105-109.

17. Chung K, Pillsbury M, Walters M, Hayward R: Reliability and validity testing of the Michigan Hand Outcomes Questionnaire. J Hand Surg [Am] 1998, 23(4):575-587

18. Adams J, Mullee M, Burridge J, Hammond A, Cooper C: Responsiveness of self-report and therapist-rated upper extremity structural impairment and functional outcome measures in early rheumatoid arthritis. Arthritis Care Res (Hoboken) 2010, 62(2):274-278.

19. Massy-Westropp N, Krishnan J, Ahern M: Comparing the AUSCAN Osteoarthritis Hand Index, Michigan Hand Outcomes Questionnaire, and Sequential Occupational Dexterity Assessment for patients with rheumatoid arthritis. J Rheumatol 2004, 31(10):1996-2001.

20. van der Giesen F, Nelissen R, Arendzen J, de Jong Z, Wolterbeek R, Vliet Vlieland T: Responsiveness of the Michigan hand outcomes questionnaire-Dutch language version in patients with rheumatoid arthritis. Arch Phys Med Rehabil 2008, 89(6):1121-1126.

21. Parsons S, Carnes D, Pincus T, Foster N, Breen A, Vogel S, Underwood M: Measuring troublesomeness of chronic pain by location. BMC Musculoskelet Disord 2006, 7:34

22. Lorig K: Outcome measures for health education and other health care interventions. Thousand Oaks: Sage Publications; 1996.

23. Brooks R: EuroQol: the current state of play. Health Policy 1996, 37(1):53-72.

24. Jenkinson C, Layte R: Development and testing of the UK SF-12 (short form health survey). J Health Serv Res Policy 1997, 2(1):14-18.

25. Norkin CC, White DJ: Measurement of joint motion: a guide to goniometry. Philadelphia: F.A. Davis; 2009

26. Ellis $B$, Bruton $A$ : A study to compare the reliability of composite finge flexion with goniometry for measurement of range of motion in the hand. Clin Rehabil 2002, 16(5):562-570

27. Kapandji A: Clinical test of apposition and counter-apposition of the thumb. Ann Chir Main 1986, 5(1):67-73.

28. Fuchs HA, Brooks RH, Callahan LF, Pincus T: A simplified twenty-eight-joint quantitative articular index in rheumatoid arthritis. Arthritis Rheum 1989, 32(5):531-537.

29. Mathiowetz V, Weber G, Kashman N, Volland G: Adult's norms for 9-hole peg test of finger dexterity. Occup Ther J Res 1985, 5:24-38.

30. Helliwell $P$, Howe A, Wright V: Functional assessment of the hand: reproducibility, acceptability, and utility of a new system for measuring strength. Ann Rheum Dis 1987, 46(3):203-208.

31. Fess E, Moran C: Clinical assessment recommendations. Philadelphia: American Society of Hand Therapists; 1981.

32. Heine PJ, Williams MA, Williamson E, Bridle C, Adams J, O'Brien A, Evans D, Lamb SE, Team S: Development and delivery of an exercise intervention for rheumatoid arthritis: strengthening and stretching for rheumatoid arthritis of the hand (SARAH) trial. Physiotherapy 2012, 98(2):121-130.

33. Vliet Vlieland TP: Non-drug care for RA--is the era of evidence-based practice approaching? Rheumatology (Oxford) 2007, 46(9):1397-1404.

34. Häkkinen A, Sokka T, Kotaniemi A, Hannonen P: A randomized two-year study of the effects of dynamic strength training on muscle strength, disease activity, functional capacity, and bone mineral density in early rheumatoid arthritis. Arthritis Rheum 2001, 44(3):515-522

35. Borg G: Psychophysical bases of perceived exertion. Med Sci Sports Exerc 1982, 14(5):377-381.

36. O'Brien AV, Jones $P$, Mullis $R$, Mulherin D, Dziedzic K: Conservative hand therapy treatments in rheumatoid arthritis-a randomized controlled trial. Rheumatology 2006, 45(5):577-583.

37. Orbell S, Sheeran P: 'Inclined abstainers': a problem for predicting health-related behaviour. Br J Soc Psychol 1998, 37(Pt 2):151-165.

38. Rejeski W, Craven T, Ettinger WJ, McFarlane M, Shumaker S: Self-efficacy and pain in disability with osteoarthritis of the knee. J Gerontol B Psychol Sci Soc Sci 1996, 51(1):P24-P29.

39. Lineker S, Badley E, Hawker G, Wilkins A: Determining sensitivity to change in outcome measures used to evaluate hydrotherapy exercise programs for people with rheumatic diseases. Arthritis Care Res 2000, 13(1):62-65. 
40. Lachin JM: Statistical considerations in the intent-to-treat principle. Control Clin Trials 2000, 21(3):167-189.

41. Moher D, Schulz K, Altman D: The CONSORT statement: revised recommendations for improving the quality of reports of parallel-group randomised trials. Lancet 2001, 357(9263):1191-1194.

42. Dunn G, Maracy M, Tomenson B: Estimating treatment effects from randomized clinical trials with noncompliance and loss to follow-up: the role of instrumental variable methods. Stat Methods Med Res 2005, 14(4):369-395.

43. Dolan P, Gudex C, Kind P, Williams A: A Social Tariff for EuroQol. York: Publications Unit, Centre for Health Economics, University of York; 1996.

44. Updated guide to the methods of technology appraisal. http://www.nice. org.uk/aboutnice/howwework/devnicetech/technologyappraisalprocessguides/ ?domedia=18mid=B52851A3-19B9-E0B5-D48284D172BD8459.

45. Unit Costs of Health and Social Care. http://www.pssru.ac.uk/uc/ uc2010contents.htm.

46. McCabe C, Claxton K, Culyer AJ: The NICE cost-effectiveness threshold: what it is and what that means. Pharmacoeconomics 2008, 26(9):733-744.

47. Charmaz K: Constructing grounded theory: a practical guide through qualitative analysis. London: Sage; 2006.

48. Smith J, Flowers P, Larkin M: Interpretative Phoneomological Analysis: theory, method and research. London: SAGE Publications; 2009:60.

49. Smith J, Flowers P, Larkin M: Interpretative Phoneomological Analysis: theory, method and research. London: SAGE Publications; 2009.

50. Smith J, Flowers P, Larkin M: Interpretative Phoneomological Analysis: theory, method and research. London: SAGE Publications; 2009:79-197.

51. Smith J, Flowers P, Larkin M: Interpretative Phoneomological Analysis: theory, method and research. London: SAGE Publications; 2009:80.

doi:10.1186/1471-2474-13-230

Cite this article as: Trial Team et al:: Strengthening and stretching for rheumatoid arthritis of the hand (SARAH): design of a randomised controlled trial of a hand and upper limb exercise intervention ISRCTN89936343. BMC Musculoskeletal Disorders 2012 13:230.

\section{Submit your next manuscript to BioMed Central and take full advantage of:}

- Convenient online submission

- Thorough peer review

- No space constraints or color figure charges

- Immediate publication on acceptance

- Inclusion in PubMed, CAS, Scopus and Google Scholar

- Research which is freely available for redistribution 